\title{
Eagle syndrome presenting with external carotid artery pseudoaneurysm
}

\author{
Anh Dao • Stellios Karnezis • John S. Lane III • \\ Roy M. Fujitani • Farhood Saremi
}

Received: 20 October 2010 / Accepted: 14 December 2010 /Published online: 7 January 2011

(C) The Author(s) 2011. This article is published with open access at Springerlink.com

\begin{abstract}
Eagle syndrome refers to a clinical syndrome caused by the abnormal elongation of the styloid process with calcification/ossification of the stylohyoid ligament. We present the first reported case of Eagle syndrome resulting in an external carotid artery (ECA) pseudoaneurysm. A patient presented to emergency room with an expanding, painful right-neck mass. CT angiography with three-dimensional volume rendering showed a bilobed $4.0-\mathrm{cm}$ right ECA pseudoaneurysm and bilateral ossification of the stylohyoid ligaments with a sharpened edge of the right styloid process at the level of the carotid artery. Aneurysmectomy was performed, and a common carotid to internal carotid bypass with reversed saphenous vein restored arterial continuity. Local resection of the styloid process with a rotational sternocleidomastoid flap was performed. The pathology report was consistent with a diagnosis of a pseudoaneurysm. A six-month clinical follow-up confirmed the complete resolution of symptoms with no neurological deficits.
\end{abstract}

\section{Introduction}

Eagle syndrome refers to a clinical syndrome caused by the abnormal elongation of the styloid process with calcification/ ossification of the stylohyoid ligament $[1,2]$. Stylo-carotid

A. Dao $\cdot$ S. Karnezis $\cdot$ F. Saremi $(\bowtie)$

Department of Radiological Sciences, U.C. Irvine Medical Center, University of California,

101 The City Drive, Route 140,

Orange, CA 92868, USA

e-mail: fsaremi@uci.edu

J. S. Lane III • R. M. Fujitani

Department of Surgery, University of California,

Irvine, CA, USA compression, typically involving the internal carotid artery, results in various symptomatic presentations. Symptoms range from pain or sensation of a foreign body in the throat to stroke and possibly even sudden death $[3,4]$. We present the first reported case of Eagle syndrome resulting in an external carotid artery (ECA) pseudoaneurysm.

\section{Case report}

A 37-year-old Hispanic male presented to the emergency room with a recently expanding, painful right-neck mass and tactile fevers. He was treated unsuccessfully with multiple courses of oral antibiotics for presumed infection. He denied significant neck trauma, intravenous drug use, or endocarditis. Laboratory exams revealed mild leukocytosis, normal erythrocyte sedimentation rate, and negative random blood cultures. CT angiography with three-dimensional (3D) volume rendering showed a bilobed $4.0-\mathrm{cm}$ right ECA pseudoaneurysm (Fig. 1) with edema extending to the skull base. CT images also showed bilateral ossification of the stylohyoid ligaments with a sharpened edge of the right styloid process at the level of the carotid artery. Preoperative angiography was performed with test balloon occlusion of the internal carotid. At operation, there was considerable inflammation of the carotid sheath, without gross purulence. Aneurysmectomy was performed with ligation of the external carotid branches. A common carotid to internal carotid bypass with reversed saphenous vein restored arterial continuity. Otolaryngology performed local resection of the styloid process with a rotational sternocleidomastoid flap. Interoperative aneurysm cultures were subsequently negative. The specimen was submitted to pathology for analysis; it consisted of a segment of a tubular structure and three fragments of a cystic structure with multiple cavities. The 
Fig. 1 Volume rendered images of $\mathrm{CT}$ angiography of the neck in right anterior oblique $(R A O)$, left anterior oblique $(L A O)$, and anterior projections demonstrate bilateral ossification of the stylohyoid ligaments. The left ligament shows an articulated form of ossification (green arrows). The right ligament is ossified at its proximal and distal sides with pencil-sharp free ends (yellow arrows). A large, 4-cm bilobed pseudoaneurysm (red arrows) has formed, arising from the origin of external carotid artery on the right. The patient felt a growing mass over the past 4 months. No history of major trauma was reported. The diagnosis was confirmed by conventional angiography. $\mathrm{ECA}=$ external carotid artery
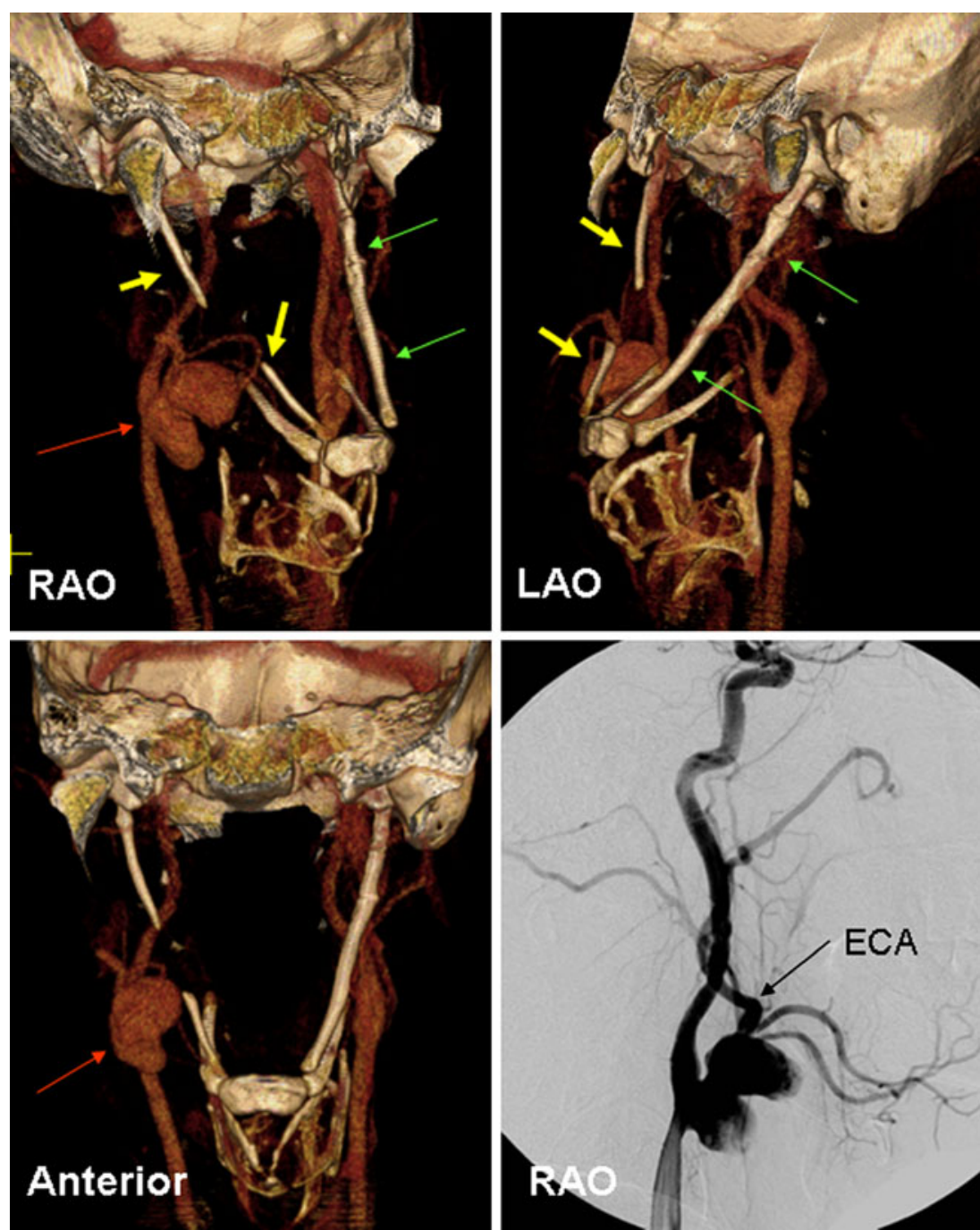

cystic structure measured $1.8 \times 1.8 \times 2 \mathrm{~cm}^{3}$ and was filled with a blood clot, diagnosed by pathology as a pseudoaneurysm. At six-month clinical follow-up, a complete resolution of the symptoms with no neurological deficits was confirmed.

\section{Discussion}

This is the first reported case of Eagle syndrome causing a pseudoaneurysm of the ECA. Eagle syndrome should be suspected in individuals with symptoms of stylo-carotid compression and can be suggested by characteristic CT findings, most pertinently a heavily ossified and calcified styloid process. CT scans can be used to determine the length of the styloid process, with recent findings suggesting 3D CT to be superior for this task [5]. The normal length of the styloid process is estimated to be $2.5 \mathrm{~cm}$, and it is generally accepted that a length greater than $3 \mathrm{~cm}$ is elongated [2]. The incidence of styloid process elongation has been estimated to be as high as $28 \%$ [6] and as low as $4 \%$ [1]. However, only a small subset of patients with an elongated styloid process ever experiences any symptoms. Eagle estimated that 4\% have symptoms while other studies have found symptoms in as many as $10 \%$ [3]. Styloid process elongation is usually bilateral, but symptoms generally appear on only one side.

There are two forms of the syndrome, both originally described by Eagle in 1937. The "classic Eagle syndrome" is usually seen in patients after tonsillectomy. Patients usually present with symptoms of ipsilateral cervicofacial pain, dysphagia, sensation of a foreign body in the throat, vertigo, tinnitus, and otalgia [3]. Additional symptoms which may manifest include a persistent sore throat, sensation of a foreign body in the pharynx, painful trismus, vocal changes, and alterations in taste [2]. The symptoms are thought to be due to impingement of cranial nerves $\mathrm{V}$, VII, IX, and X by the elongated styloid process [3].

The second form of the syndrome is attributed to impingement of the internal or external carotid artery by a laterally or medially deviated styloid process. Pain can occur as a result of stimulation of the sympathetic nerve 
plexus associated with the artery. When impingement of the internal carotid artery occurs, pain is felt along the course of the artery and may be accompanied by pain in the eye and parietal cephalgia, which can lead to incorrect diagnoses such as cluster headache or migraine [3]. Impingement of the external carotid artery leads to pain in the face below the eyes [3].

Neurological symptoms can also occur when there is an interruption of blood flow to areas that the internal or external carotid artery supplies. There have been case reports of patients with an elongated styloid process who suffered from transient ischemic attacks on turning their head, only to resolve once their head was returned to the neutral position $[3,4]$. These patients had symptoms such as aphasia, visual disturbances, weakness, and syncope. Imaging studies showed focal flow restriction. Surgical resection in these cases led to a complete resolution of the symptoms. Rarely, compression of both carotid sinuses by bilaterally elongated styloid processes can occur, potentially even leading to sudden death from vagus-mediated cardiac inhibition [6].

Impingement of the carotid arteries by an elongated styloid process can also lead to direct mechanical damage and the development of a carotid dissection [7]. Additionally, there has been a report of distal extracranial internal carotid artery aneurysm developing in a patient with a prominent styloid process. Angiograms demonstrated the styloid process directly impinging on the aneurismal sac, with a speculation that repetitive trauma may have resulted in aneurysm formation [8]. External carotid artery pseudoaneurysm from hyoid bone fracture is also reported [9]. However, no prior reports describe the development of an external carotid artery pseudoaneurysm as a result of Eagle syndrome. It is conceivable that these are all related processes that occur because of traumatic injury to the carotid artery from the elongated styloid process. As a result of the traumatic injury to the carotid artery, patients may develop a carotid dissection, an aneurysm, or a pseudoaneurysm. Future studies are necessary to investigate the relationship between an elongated styloid process and the development of these complications.

Open Access This article is distributed under the terms of the Creative Commons Attribution Noncommercial License which permits any noncommercial use, distribution, and reproduction in any medium, provided the original author(s) and source are credited.

\section{References}

1. Eagle WW (1949) Symptomatic elongated styloid process: report of two cases of styloid process-carotid artery syndrome with operation. Arch Otolaryngol 49:490-503

2. Piagkou M, Anagnostopoulou S, Kouladouros K et al (2009) Eagle's syndrome: a review of the literature. Clin Anat 22:545-558

3. Chuang WC, Short JH, McKinney AM et al (2007) Reversible left hemispheric ischemia secondary to carotid compression in Eagle syndrome: surgical and CT angiographic correlation. Am J Neuroradiol 28:143-145

4. Farhat HI, Elhammady MS, Ziayee H et al (2009) Eagle syndrome as a cause of transient ischemic attacks. J Neurosurg 110(1):90-93

5. Savranlar A, Lokman U, Mehmet BU et al (2005) Threedimensional CT of Eagle's syndrome. Diagn Interv Radiol 11:206-209

6. Runwanpura PR, Abeygunasekera AM, Tikiri GK (2008) Sudden unexpected death probably due to Eagle's syndrome: a case report. Med Sci Law 48(4):350-353

7. Zuber M, Meder JF, Mas JL (1999) Carotid artery dissection due to elongated styloid process. Neurology 53(8):1886-1887

8. Sundt TM, Pearson BW, Piepgras DG et al (1986) Surgical management of aneurysms of the distal extracranial internal carotid artery. J Neurosurg 64:169-182

9. Campbell AS, Butler AP, Grandas OH (2003) A case of external carotid artery pseudoaneurysm from hyoid bone fracture. Am Surg 69(6):534-535 